\title{
Design strategies of an unobscured three mirror telescope with freeform surfaces for infrared nanosatellite imagery
}

Louis Duveau, Thierry Lépine, Emmanuel Hugot, Xavier Briottet, Guillaume Druart

Louis Duveau, Thierry Lépine, Emmanuel Hugot, Xavier Briottet, Guillaume Druart, "Design strategies of an unobscured three mirror telescope with freeform surfaces for infrared nanosatellite imagery," Proc. SPIE 11852, International Conference on Space Optics - ICSO 2020, 118520C (11 June 2021); doi: 10.1117/12.2599153

SPIE Event: International Conference on Space Optics - ICSO 2021, 2021, Online Only 


\section{International Conference on Space Optics-ICSO 2020}

Virtual Conference

30 March-2 April 2021

Edited by Bruno Cugny, Zoran Sodnik, and Nikos Karafolas
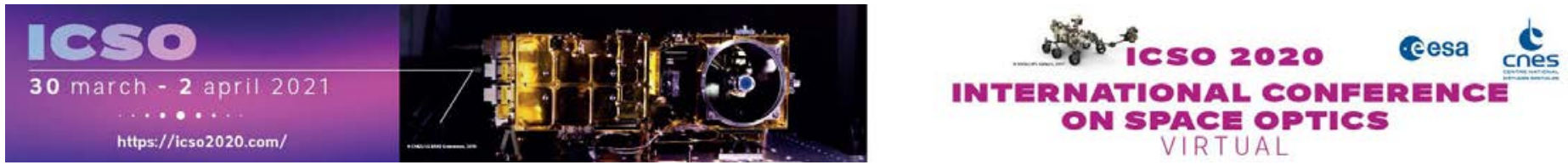

\section{Design strategies of an unobscured three mirror telescope with freeform surfaces for infrared nanosatellite imagery}

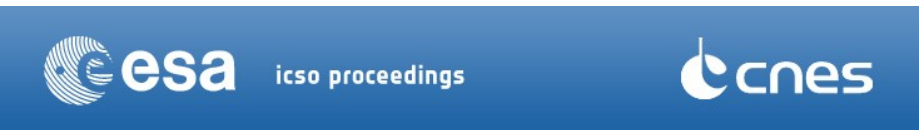




\title{
Design strategies of an unobscured three mirror telescope with freeform surfaces for infrared nanosatellite imagery
}

\author{
Louis Duveau ${ }^{\mathrm{a}}$, Thierry Lépine ${ }^{\mathrm{b}}$, Emmanuel Hugot $^{\mathrm{c}}$, Xavier Briottet $^{\mathrm{a}}$, and Guillaume Druart ${ }^{\mathrm{a}}$

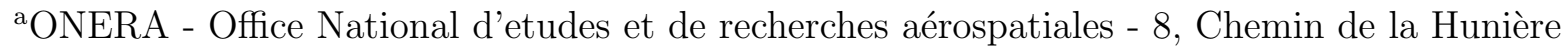 \\ BP80100 91123 PALAISEAU CEDEX France \\ ${ }^{\mathrm{b}}$ Univ Lyon, Laboratory Hubert Curien, CNRS UMR 5516 - 18, Rue du Professeur Benoît \\ Lauras 42000 Saint-Etienne France \\ cAix-Marseille Univ., CNRS, CNES, LAM - 38, rue Fredéric Joliot-Curie 13388 Marseille cedex \\ 13 France
}

\begin{abstract}
The new degrees of freedom offered by the new family of freeform surfaces helps compensating for off axis aberrations in optical designs. We define a freeform surface as a non-rotationally symmetric surface which cannot be described as an off-axis part of a conicoid. The use of freeform surfaces allows an increase in performance such as on the field of view, the F-number and compactness of unobscured all reflective telescopes. Reflective designs have the advantage of being light, achromatic, and potentially low-cost due to cheaper materials involved. It could result in industrial production of compact panchromatic systems for various applications ranging from automotive sensors to imaging nanosatellites. In this paper we address the challenges posed by the use of these surfaces in optical design optimization, specifically the minimization of the surface counting, in order to simplify the manufacturing, assembly and integration phases. We focus on three mirror designs and work on the compactness with various flavors in the 3D space.

We will discuss our results on a fast, unobscured, non reimaging and well corrected three mirror anastigmat (TMA) design for a nanosatellite thermal imaging payload using uncooled bolometers. Optimization method of a TMA under volume and straylight constraints is described. A tolerance analysis is performed and minimal alignment is required even for large tolerances.
\end{abstract}

Keywords: freeform, optical design, nanosatellite, TMA, infrared imaging, straylight

\section{INTRODUCTION}

Optical design has dramatically changed in the past decades. The use of computer assisted optimization allowed designers to perform fast and precise computation allowing them to imagine complex optical systems. Imaging optical systems became better corrected due to the capability for designers to study systems with an increasing number of parameters, and thus allowed them to use more optical surfaces, and the possibility to fine tune all of these parameters using computational optimization process. The idea of using non rotationally symmetric optical surfaces is not new. In a generic way, a freeform surface is sometimes considered as a surface with an unconstrained shape. However, to differentiate this type of surface from others, we will consider that a freeform surface is a surface that cannot be defined as a subaperture of a rotationnaly symmetric surface. This exclude aspheres and off centered sub pupils of aspheric surfaces. In this article, we present a freeform TMA with a rectangular FOV for multispectral infrared imaging. It is a preliminary design for a space constellation of nanosatellite to study the urban heat island phenomenon.

The Urban Heat Island (UHI) is a phenomenon that most people are aware of. In urban areas, the intense human activity and energy consumed in a small area and the use of materials with a low albedo and high volumetric heat capacity increases this effect. The energy used to cool down buildings only add up to the problem. ${ }^{1}$ To measure those effects, both on-site and remote data collection of temperature can be used. However,

Further author information: (Send correspondence to Louis Duveau)

Louis Duveau: E-mail: louis.duveau@onera.fr 
to overcome the sparse spatial sampling of on-site data, the use of remote temperature measurements seems to be the solution. It is possible to use data collected by satellites such as the Landsat satellites. ${ }^{2}$ These satellites are placed in sun-synchronous orbits* ${ }^{*}$, with long revisit periods (16 days for Landsat mission satellites). Such low revisit always at the same solar time does not give a sufficient amount of data to analyze properly the UHI. To increase the revisit frequency up to a few measurements per day, which is necessary to sample the circadian cycle, the number of satellites must be increased dramatically. ${ }^{3}$ However, such satellites embark high performance but expensive payloads and a constellation of this type of satellites is not financially reasonable.

To keep a good spatial sampling combined with a high revisit frequency, the use of multiple satellites in a constellation is mandatory but at a low cost and at a low mass. Therefore cubesats, which are small satellites of only a few cubic decimeters, are getting more and more popular for such scientific missions. ${ }^{4}$ Such satellites can be mass-produced for a reasonable price. However, it implies the manufacturing of ultra compact optical payloads, which is possible using freeform surfaces.

\section{SPECIFICATIONS}

Here are the principal requirements for the optical payload:

1. The payload must provide images in the thermal infrared spectrum. The measurement must be done in several spectral band to correct atmospheric and emissivity effects in order to retrieve the Land Surface Temperature. A minimum of three spectral band is required to measure land emissivity and thus correctly estimate the land surface temperature.

2. The spatial resolution must be between 50 to $100 \mathrm{~m}$ and the swath between 50 to $100 \mathrm{~km}$.

3. The whole satellite must be a $12 \mathrm{U}$ cubesat in low earth orbit altitude.

In order to keep a low cost, compact and easy to manufacture solution, we will study a design with an uncooled microbolometer and an off-axis unobscured telescope. ${ }^{5}$ The use of a fully reflective design allows to consider for multispectral imaging by using a spectral separation after the last mirror. Indeed mirrors allows for an achromatic solution, and for an athermic solution if the whole instrument is made in the same material. Moreover mirrors are lighter than lenses and infrared optical materials are expensive. To produce an image of the scene with such a satellite, using a pushbroom ${ }^{\dagger}$ technique is very useful to have a simple and compact instrument. As presented in $^{6}$ and in figure 1 a two mirror freeform telescope is sufficient for this kind of mono-dimensional FOV.

However in this project, we need to have several spectral bands in the infrared (IR) spectrum. We also need to increase the time during wich a ground pixel is seen by the optical system, in order to increase the signal to noise ratio (SNR) by using post-processing Time Delay and Integration (TDI). To this end, we will increase the field of view of the system and use a full SXGA matrix size of a microbolometer with a pixel pitch of $12 \mu \mathrm{m}$. Using the whole detector will allow for the use of several filters and thus have a multispectral instrument in the thermal spectrum(also called long-wave infrared (LWIR)) as illustrated in figure 2. Using an off axis three mirror anastigmat (TMA) telescope for such a design is quite common. ${ }^{7,8}$ With an uncooled infrared detector, the system must have a low F number, under 1.5, to keep an acceptable NETD ${ }^{\ddagger}$.

The spatial resolution is limited by the pixel size, which has an instantaneous field of view (IFOV) depending on the focal length of the instrument. Knowing the altitude, this IFOV can be expressed as dimensions of visible ground area, or ground sampling distance (GSD). The optical system modulation transfer function is also an important indicator to ensure that the Nyquist frequency of the detector is actually resolved. For this design we

\footnotetext{
*orbit around the earth in which the satellite always passes over any given point of the surface of the planet at the same local mean solar time

${ }^{\dagger}$ The movement of the satellite above the scene allows to produce a two dimensional image with a mono-dimensional field of view of the optical system, orthogonal to the movement

${ }^{\ddagger}$ noise equivalent temperature difference
} 


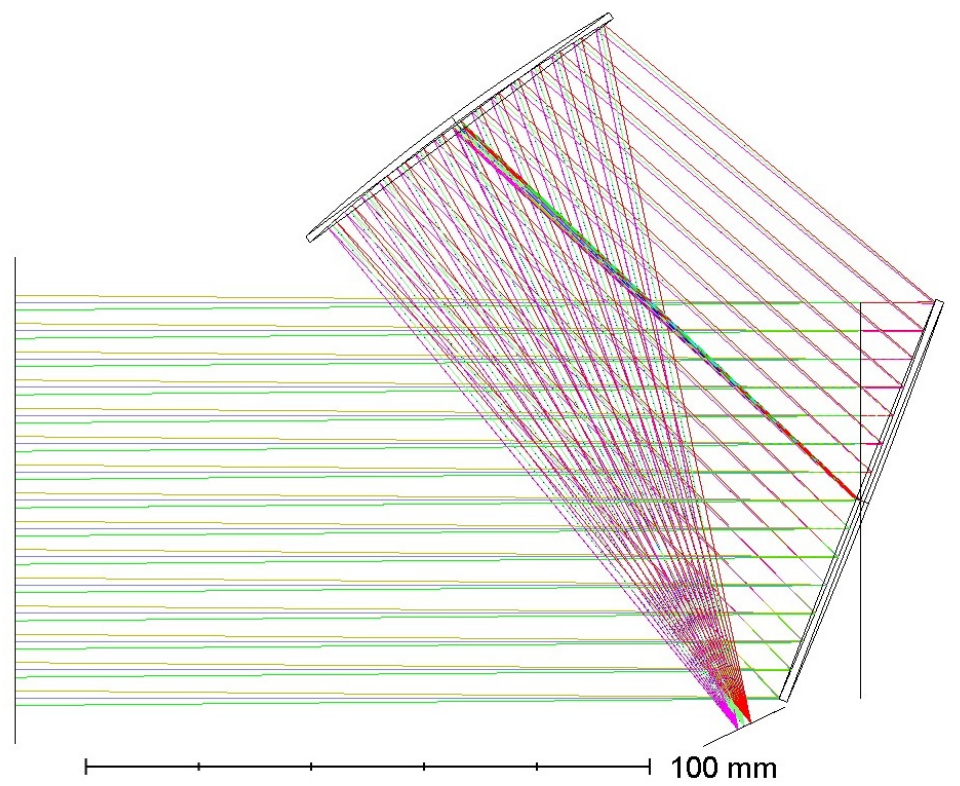

Figure 1: Example of a two mirror freeform telescope for pushbroom applications $\mathrm{F} \#: 2.1$, f:150mm, fields: $1^{\circ} Y$ $\& 6^{\circ} X$

chose a 100m GSD, compliant with the requirements. The orbits have been calculated to be around $570 \mathrm{~km}$ with a small eccentricity. The focal length can be deduced:

$$
f=\frac{\text { mean altitude } * \text { pixel size }}{G S D} \approx 70 \mathrm{~mm}
$$

But the image also suffer from motion blur due to the satellite movement above the ground. The mean orbital speed of this satellite would be:

$$
v_{0}=\sqrt{\frac{\mu}{a}} \approx 7,6 \mathrm{~km} / \mathrm{s}
$$

with $\mu$ the standard gravitational parameter and $a$ the semimajor axis of the orbit.

This motion blur causes a loss in high frequency content and thus in resolution. To minimize the motion blur, the motion of the satellite in respect to the ground must be less than the GSD during the acquisition of an image. For a GSD of $100 \mathrm{~m}$ and a displacement of $7,6 \mathrm{~km} / \mathrm{s}$, the ideal framerate would be $76 \mathrm{~Hz}$. In this paper, we anticipate that the technology could be available in a near future, as SXGA uncooled detectors with a framerate of $60 \mathrm{~Hz}$ already exists. However, the design presented can also be adapted for a bigger GSD (using a shorter focal length) with a higher aperture and slower framerate, trading spatial resolution for radiometric accuracy.

The half field of view (half FOV) along each direction when using a SXDA detector is:

$$
\begin{array}{r}
\text { half } F O V=\tan ^{-1}\left(\frac{\text { half } \text { image size }}{f}\right) \\
\text { half } F O V X \approx 6,25^{\circ} \\
\text { half } F O V Y \approx 5^{\circ}
\end{array}
$$

Finally, The whole $12 \mathrm{U}$ must be shared between the optical system, electronics and the platform subsystems, and we therefore cannot allocate all volume for the optical payload, and set a limit of $8 \mathrm{U}$ allocated to the optical payload itself. We will choose an allocated volume of $100 \times 200 \times 300 \mathrm{~mm}^{3}$, which is a $6 \mathrm{U}$ volume. To account for the mirrors substrate, mechanical design and detector electronics, we will allow a $100 \times 150 \times 250 \mathrm{~mm}^{3}$ volume for the optical design itself. Table 1 and figure 2 summarize the specifications of the optical payload. 


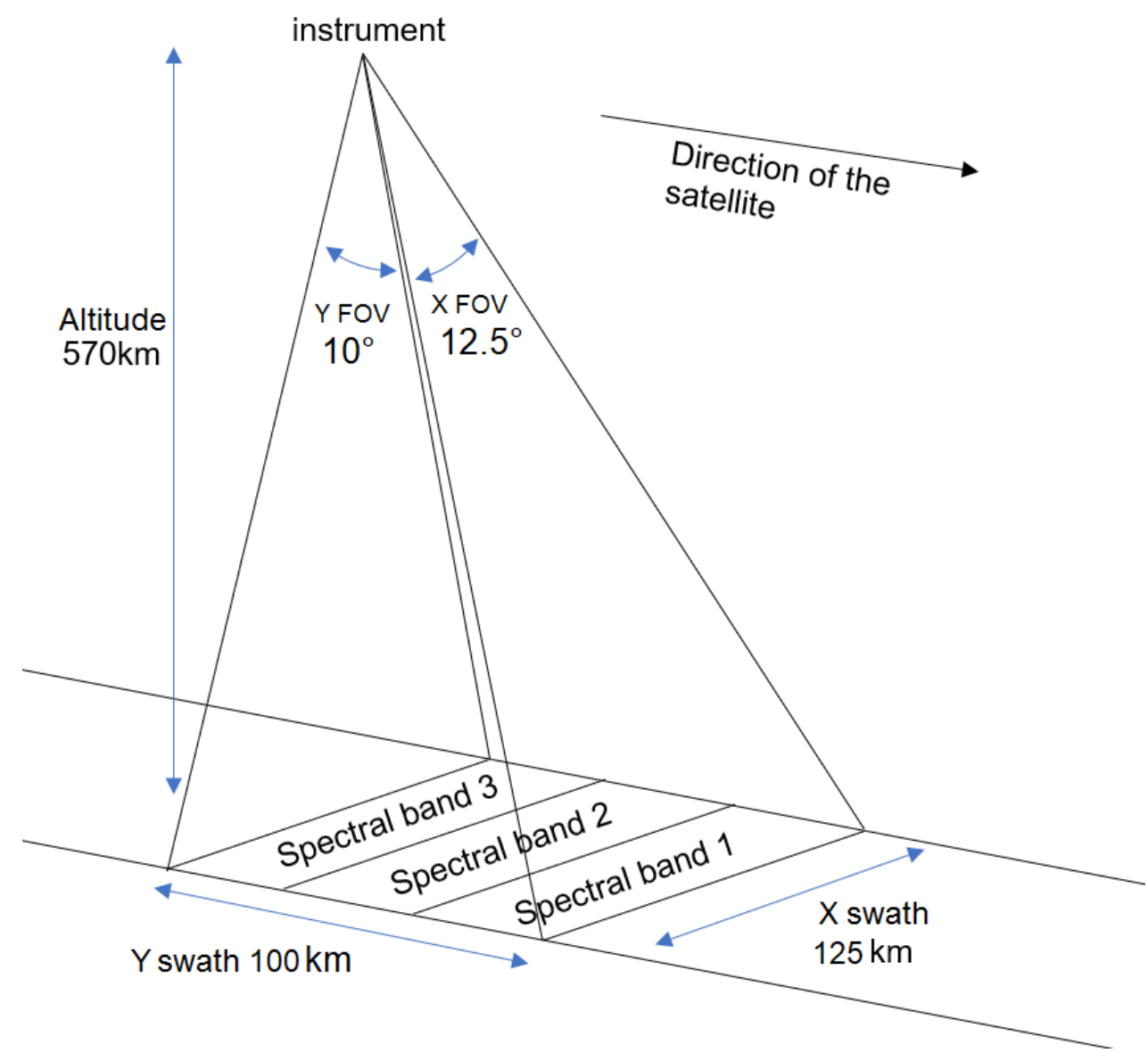

Figure 2: Summary of specifications and illustration of the field division for the multispectral imaging

Table 1: Summary of specifications

\begin{tabular}{cc}
\hline specification & value \\
\hline effective focal length & $70 \mathrm{~mm}$ \\
half FOV $X$ & $6,25^{\circ}$ \\
half FOV $Y$ & $5^{\circ}$ \\
F\# & 1.5 \\
pixel size & $12 \mu \mathrm{m}$ \\
number of pixels & $1280 X^{*} 1024 Y$ \\
max $X$ dimension & $100 \mathrm{~mm}$ \\
$\max Y$ dimension & $250 \mathrm{~mm}$ \\
$\max Z$ dimension & $150 \mathrm{~mm}$ \\
\hline
\end{tabular}




\section{FREEFORM OPTICS}

Creating designs using freeform surfaces implies to be able to use a very high number of parameters and have precise ray tracing algorithms, due to the number of parameters required to define the surface shapes. Rotationnaly symmetric optical design allows the designer to work only on a cross section of the design while freeform design is inherently a 3D process, which demands a lot more computational power and explains that it is only a recent trend in optical design. Continuous progress in computational power and algorithms made freeform designs possible to optimize and freeform manufacturing techniques make these concepts realizable. Designers are left with an entire new family of systems to explore and conceptualize. In this family of new systems, we will focus on the group of short focal length catoptric designs with large field of view (FOV). Classical on axis catoptric designs are used for long focal lengths and small FOV because symmetry eases the design, manufacturing and test of surfaces. But on axis reflective systems suffer from central obscuration that scales with the FOV, usually due to an increase in the size of the secondary mirror, enventually until central obscuration reaches $100 \%$. To cope with this issue, unobscured off axis systems have been designed without using freeform surfaces. However, these systems still usually have a small FOV. Indeed, decentering surfaces add some new kind of geometrical aberrations. These assymetric aberrations augment with the mirrors tilts and decenters, which are linked with apeture and FOV, therefore being harder to compensate while keeping rotationnaly symmetric surfaces. ${ }^{9}$ The use of freeform surfaces allows for a better compensation of those nodal aberrations and enables large FOV designs. To emphasize the interest of using freeform surfaces, we will try to minimize the surface counting to have the simplest design possible with minimal alignment and manufacturing costs. To summarize, the use of freeform surfaces allows an increase in performance such as the field of view, the F-number, and the suppression of obscuration on compact all-reflective systems. ${ }^{10,11}$ This allows compact and well corrected off axis reflective designs. Reflective designs have the advantage of being light, achromatic, and potentially low-cost due to cheaper materials involved. It could result in industrial production of compact panchromatic systems for various applications ranging from automotive sensors to imaging cubesat. ${ }^{12}$

Freeform surfaces are mostly defined as a standard conic surface with a 2D polynomial correction, mostly over polar coordinates, ${ }^{11}$ but many other definitions exist, as Splines, Nurbs or radial basis functions ${ }^{\S} .{ }^{13}$ Article ${ }^{14}$ gives an overview of standard freeform surfaces description.

The two polynomial basis we will focus on are the Zernike polynomials and the XY polynomials:

$$
\begin{gathered}
\text { Zernike }: z(r, \varphi)=\frac{c r^{2}}{1+\sqrt{1-(1+k) c^{2} r^{2}}}+\sum_{i=1}^{N} A_{i} Z_{i}(\rho, \varphi) \\
X Y: z(x, y)=\frac{c r^{2}}{1+\sqrt{1-(1+k) c^{2} r^{2}}}+\sum_{i=1}^{N} A_{i} E_{i}(x, y)
\end{gathered}
$$

$c$ is the curvature of the surface, $k$ is the conic constant, $Z_{i}$ is the $i$ th Zernike polynomial, and $E_{i}$ is the $i$ th $\mathrm{XY}$ polynomial.

Every surface is then described by many coefficients instead of only 2 for a conic surface. This complexity is however overcame thanks to the high computational power of modern computers that can optimize on a large number of parameters, even if the optimization problem is a very complex issue that is studied by many researchers worldwide in numerous fields.

The most used definition is the normalized Zernike basis because it is an orthonormal basis over the unit disc. With a proper normalization, it is possible to have a unique description of the surface on the Zernike polynomial basis. Moreover, the Zernike polynomials are used to describe aberrations and it is often useful to look at the wavefront and the surfaces shapes with the same basis. However, the use of XY polynomials is very convenient in the early stages of optimization.

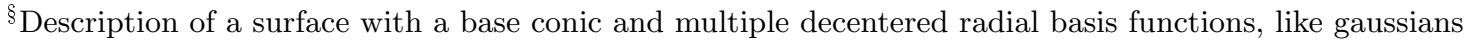




\section{OPTICAL DESIGN AND ANALYSES}

\section{1 optical design}

This optical system is designed with three freeform mirrors defined with polynomial bases. As the mirrors are tilted, allowing tilt to the surface would cause a degeneracy that could have a negative effect on the optimization process. ${ }^{15}$ Moreover, removing any local tilt of the surface at its center allow for a complete control on the path of the parabasal ray and surface positioning. To ease this design process, we initially designed the system using $\mathrm{XY}$ polynomials with null terms coefficients for the X and Y monomials. This initial optimization is performed with polynomials up to the fifth order. It is interesting to note that the construction of Zernike polynomials and XY polynomials is such that for any given order both bases describe the same subspace of surfaces (piston excluded). Using this property, an analytical conversion from XY to Zernike is performed. Another optimization using Zernike polynomials is performed up to the seventh order. Local tilt is also cancelled using macros and the coefficient associated with the focus term is also kept at zero to avoid degeneracy with the base sphere. A standard optical design software have been used, while avoiding using any functions that use paraxial data. As an example the focal length, being a paraxial value, is not relevant anymore. The focal length and distortion are kept by ensuring the same magnification as a $70 \mathrm{~mm}$ classical design and this magnification is kept over the field by fixing the real image position and shape. The result is the TMA visible in the figure 3 .

Several systems have been studied, and placing the stop on the third mirror gave the best compactness. For smaller FOV, it is interesting to note that placing the stop on the secondary mirror seems to be empirically better.

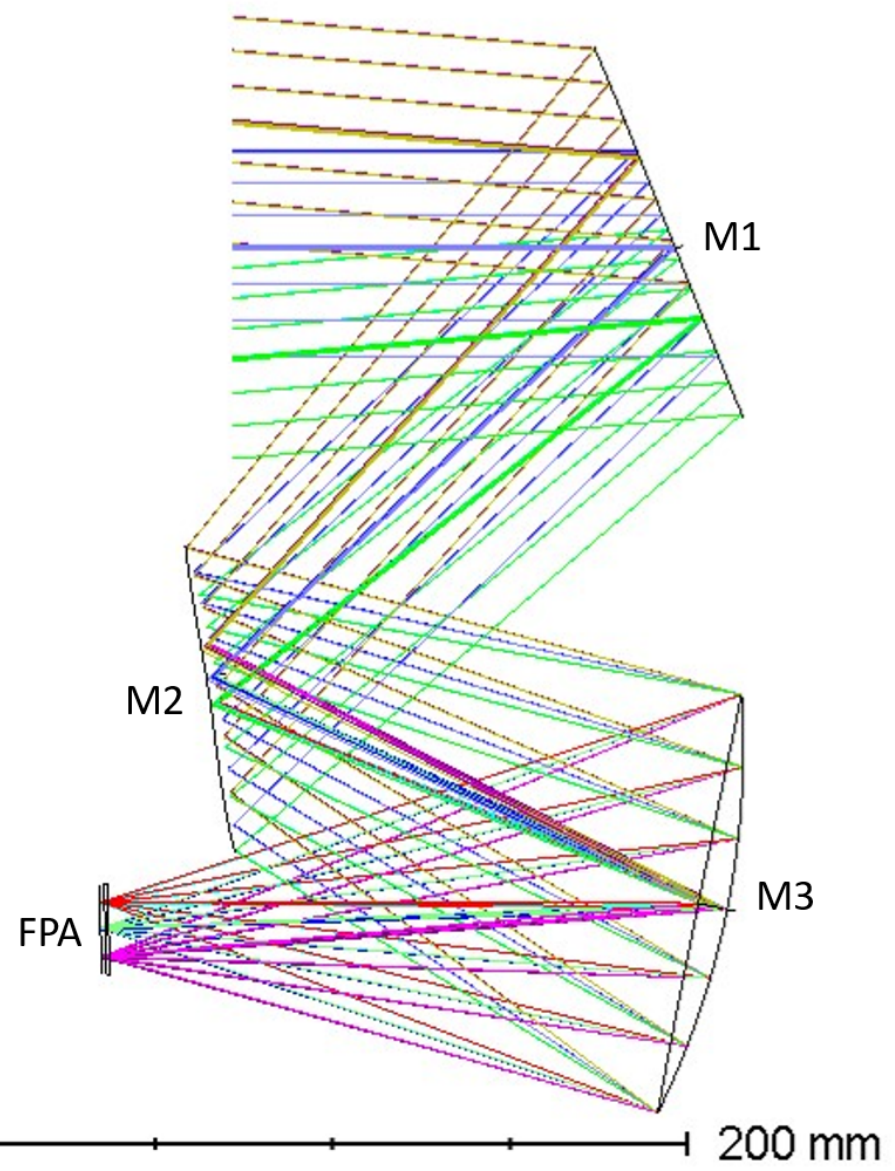

Figure 3: Layout of the optical system, FPA: Focal plane array 
The performances are visible in the figures 4 and 5 . The optical system is diffraction limited for a $10 \mu \mathrm{m}$ wavelength.

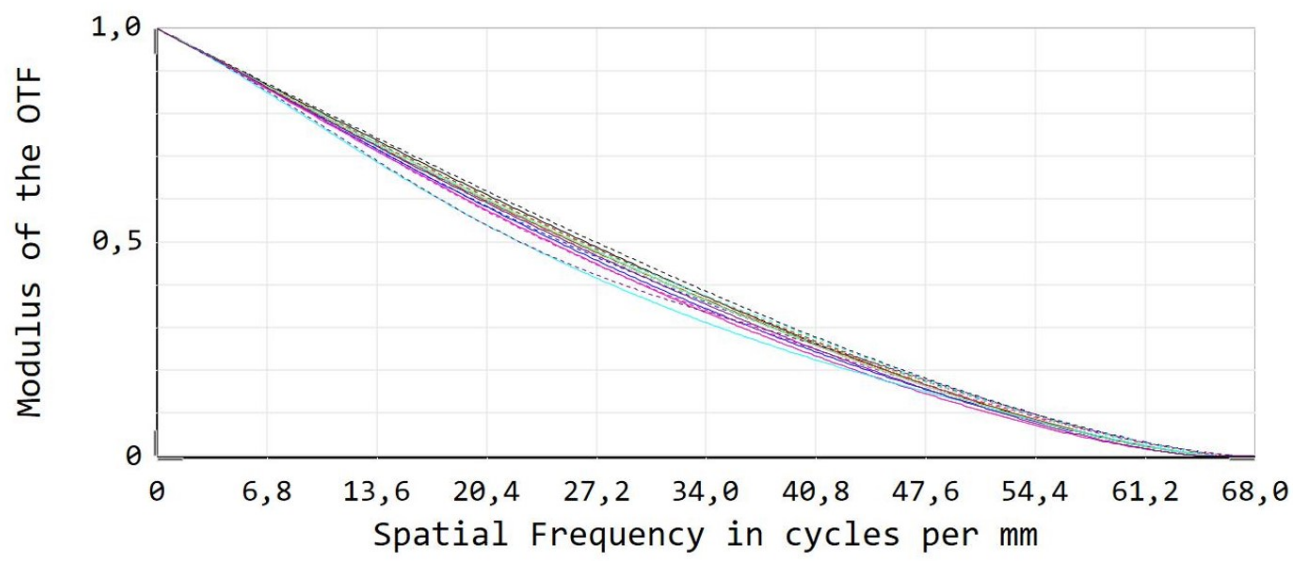

Figure 4: Modulation transfer function at $10 \mu \mathrm{m}$

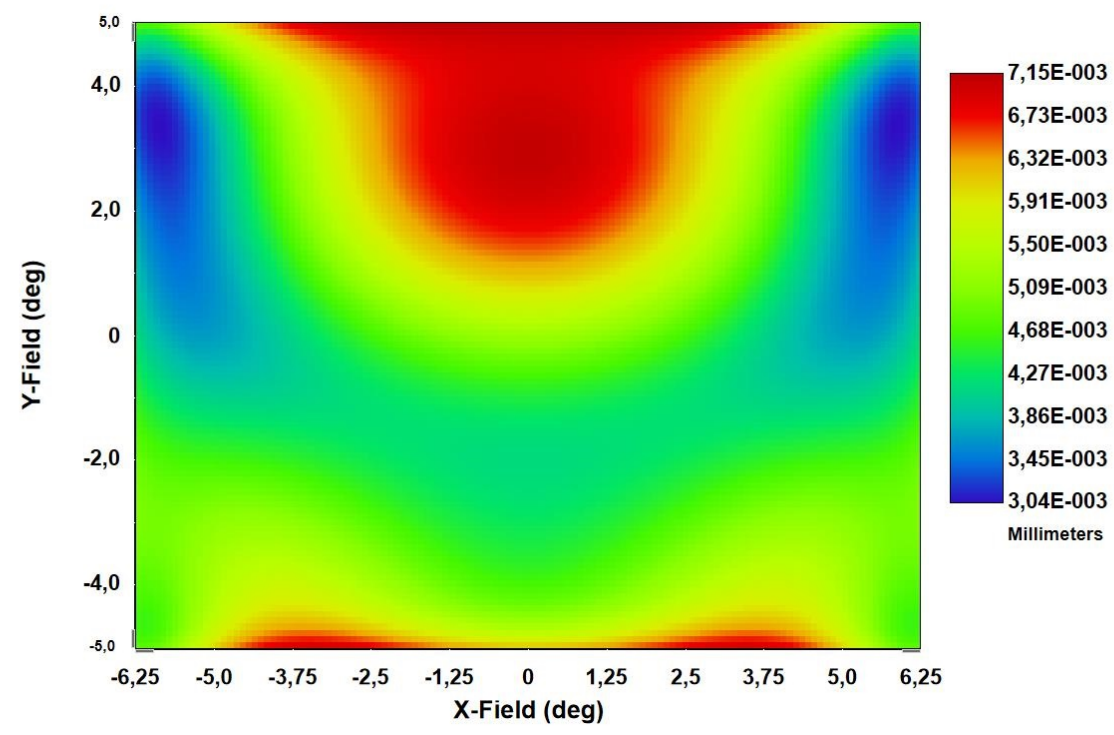

Figure 5: RMS spot radius over the field. Airy radius $=18,3 \mu \mathrm{m}$ Min RMS spot radius $=3,04 \mu \mathrm{m}$ Max RMS spot radius $=7,15 \mu \mathrm{m}$

\section{2 manufacturing and tolerancing}

This system is intended to be manufacturable. To assess the difficulty to manufacture the mirrors, the departures from the best spheres for each mirror are pictured on figures 6, 7 and 8. To account for turning manufacturability, the orthoradial slopes are aslo pictured. Only the useful portion of each mirror is shown even though the optimization is performed on a circular basis shape.

Surfaces that are shown here only have a few millimeters sag and orthoradial slopes are only a few degrees. Those values are in the range of diamond turning machines capabilities. ${ }^{16}$ The main drawback of having large 

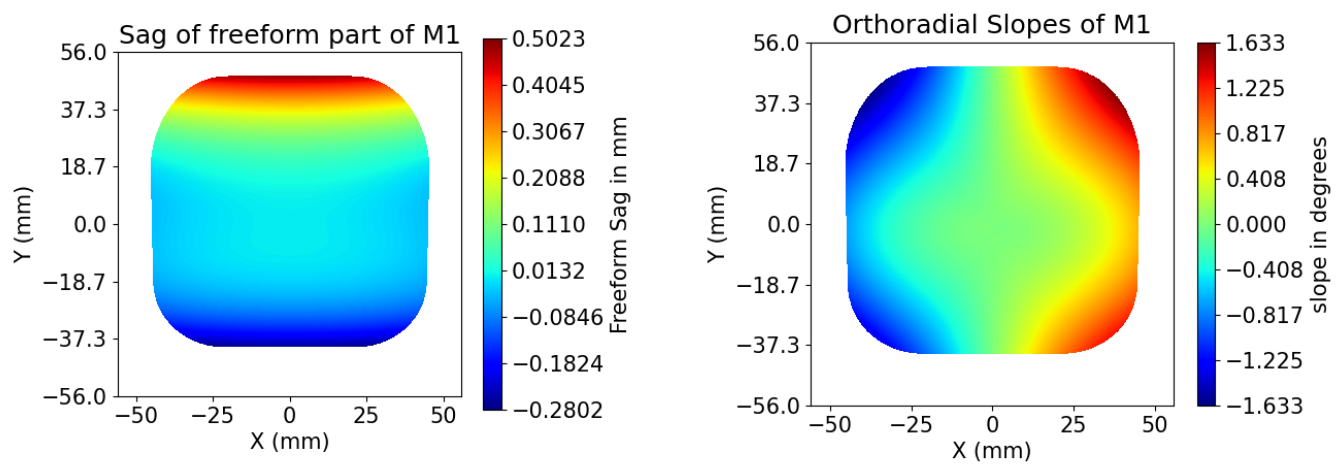

Figure 6: Left: Residual sag of the M1 after removal of the base radius. Right: Orthoradial slopes of the M1.
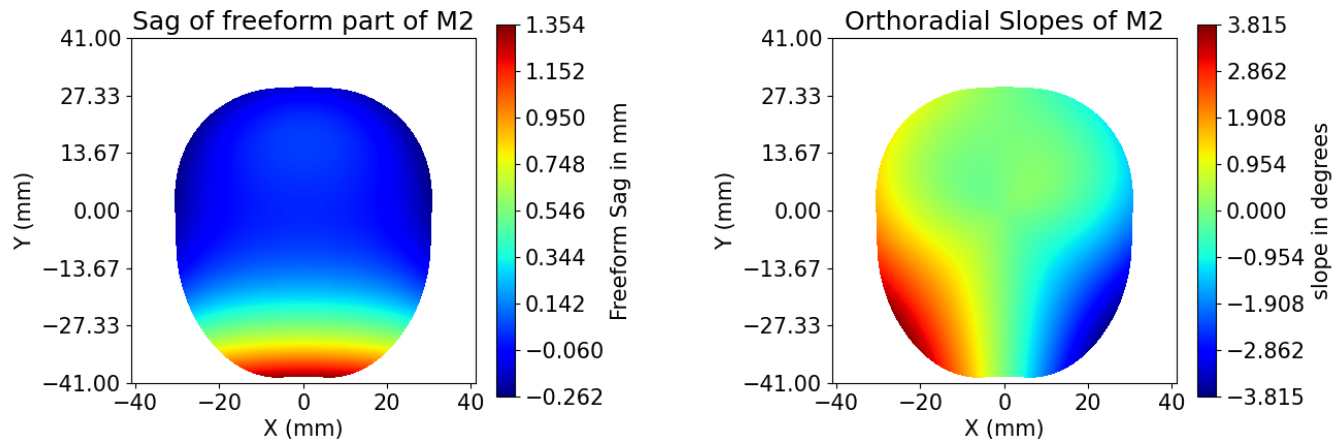

Figure 7: Left: Residual sag of the M2 after removal of the base radius. Right: Orthoradial slopes of the M2.
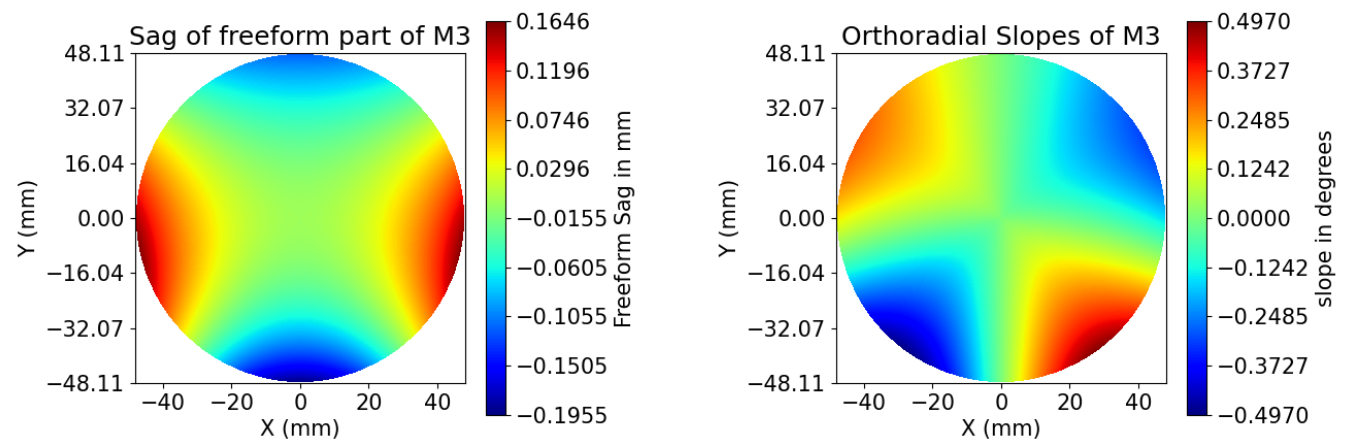

Figure 8: Left: Residual sag of the M3 after removal of the base radius. Right: Orthoradial slopes of the M3. 
FOV is that the freeform departure on the M2 is very high and this could complicate metrology measurements. However, there a many ways to perform metrology other than interferometry that are quickly improving. ${ }^{17}$

To ensure that the system is manufacturable, manufacturability of each surface is not sufficient. In this work, we performed a tolerance analysis of the system. The tolerances are given in Table 2 and the compensators are the focus and the tip/tilt of the third mirror.

Table 2: Summary of tolerances

\begin{tabular}{cc}
\hline parameter & value \\
\hline Position of the mirrors & $100 \mu \mathrm{m}(\mathrm{X}, \mathrm{Y}, \mathrm{Z})$ \\
Tilt of the mirrors & $1 \mathrm{mrad}$ \\
Tilt of the focal plane & $0.1 \mathrm{degree}$ \\
\hline
\end{tabular}

A Monte Carlo approach has been used to analyse the toleranced performance of this system. A total of one thousand systems have been generated using a normal distribution. The results, given in Table 3, show without any doubt that such systems can be manufactured with large tolerances if an alignment of the third mirror is allowed.

Table 3: Tolerancing results

\begin{tabular}{cc}
\hline Nominal RMS spot radius (field averaged) & $5,15 \mu \mathrm{m}$ \\
Mean RMS spot radius & $6,00 \mu \mathrm{m}$ \\
standard deviation & $0,52 \mu \mathrm{m}$ \\
\hline M3 tilt X standard deviation & 0,028 degrees \\
M3 tilt Y standard deviation & 0,019 degrees \\
focus standard deviation & $30 \mu \mathrm{m}$ \\
\hline
\end{tabular}

\section{3 straylight analysis}

The accuracy of the temperature measurement is limited by the noise-equivalent temperature difference (NETD). This NETD is driven by the signal to noise ratio of the instrument. As uncooled microbolometers used as fast frequency (a few dozens of $\mathrm{Hz}$ ) have a low sensitivity and a high thermal noise, the signal needed is high and therefore the F number must be kept as low as possible. This is also useful to increase the diffraction limit of the system and therefore the MTF at higher spatial frequencies.

The residual fixed pattern noise (RFPN) is also problematic. Microbolometers suffer from high fixed pattern noise, which must be kept under the NETD. To correct this effect, infrared systems are carefully calibrated before launch. However, there is a drift in this noise and thus the correction must be updated regularly using cross calibration with data collected by reference satellites.

Finally, the last source of error is the straylight variation. As the focal plane array is uncooled, so is the rest of the satellite. Every part located in front of the detector will emit infrared straylight and perturb the measurement. This straylight is not critical as long as it stays stable (or predictable) over time. The straylight added by the system itself depends on its temperature, so this aspect must be undestood and controlled to maintain both optical and radiometric performances. The out of field straylight is however unpredictable. If such parasite light reaches the detector, it means that the unknown out of field temperature modifies the temperature measurement inside the FOV of the instrument. This implies that out of field straylight must be as limited as possible by design.

For this design, a specific set of constraints have been implemented to nullify the specular out of field straylight. Specular straylight is the straylight of an idealized system where mirrors have a reflection factor of one, and all the other parts absorb all incident light, without taking into account diffusion or emission by the surfaces. The constraints used are the following: 


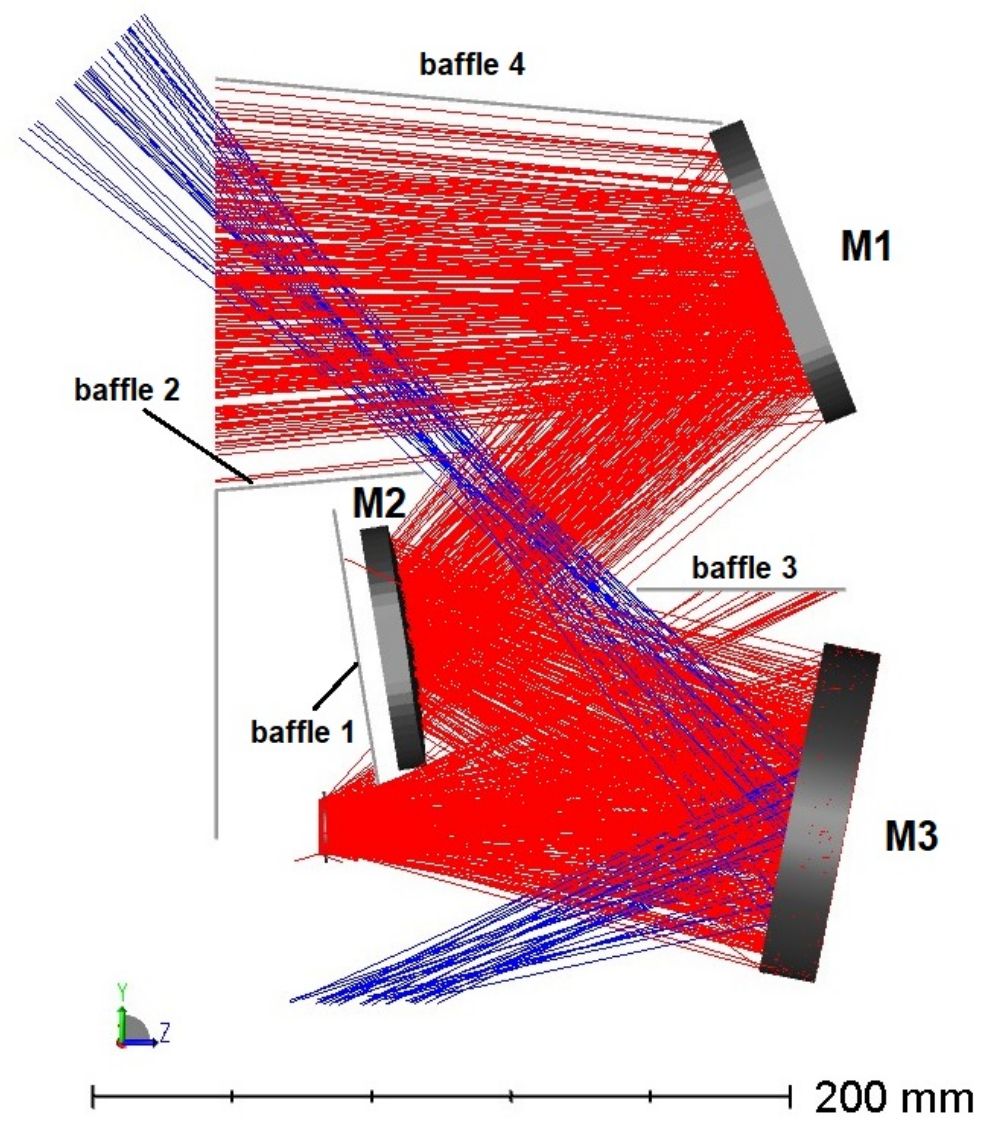

Figure 9: Straylight analysis summary of the optical system

1. no ray must reach the detector without being reflected on the third mirror. This is reached by ensuring that the detector can only see the M3 or baffles.

2. no ray can reach the third mirror without being first reflected on the first and second mirrors or without having an high angle of incidence ensuring that it gets reflected on a surface far from the detector that can be hidden from it.

3. as any ray reaching the detector must be reflected by all three mirrors, optical properties ensure that if it hits the detector it is a ray from inside the FOV of the instrument.

The first constraint is achieved using the placement of the detector behind a baffle behind the secondary mirror (Baffle 1 in 9. In practice this baffle could be fixed on the secondary mirror support. As the detector is placed behind the secondary, no light can reach the detector from this mirror either. A horizontal baffle (baffle 2) prevent any ray going straight from the object space to the detector. The result is that the only optical element seen from the detector is the third mirror. The second constraint is achieved using baffle 2, and another horizontal baffle (baffle 3): only rays that are far out of field can reach the third mirror. These rays with a high angle of incidence on the third mirror are reflected far from the detector, in the bottom of the satellite (they are represented in blue in the figure 9). A baffling on the entrance aperture (baffle 4) limit the amount of those straylight rays reaching the inside of the system. A longer baffle could be used to block more of those rays. The straylight analysis is summarized on the figure 9. The figure displays a non sequential ray tracing on the system, with the three added baffles. two sources have been placed: 
1. a quasi lambertian source on the detector for the red rays.

2. a quasi-lambertian source out of field for the blue rays.

Red rays are used to analyse potential straylight paths that reach the detector. The fact that all rays coming out of the system are within the FOV and reflected only on the three mirrors ensure that there is no specular straylight path reaching the detector.

\section{CONCLUSION}

In this article we presented a practical example of a lightweight infrared or multi-spectral instrument freeform optical design for a $12 \mathrm{U}$ nanosatellite to perform land temperature measurements of urban heat islands. The design is based on a high aperture unobscured freeform TMA without specular straylight and an uncooled microbolometer. The performances are also ensured to be satisfying with minimal alignment. The design and tools used to optimize it have been kept versatile enough to ensure further optimization taking manufacturer capabilities into account or missions modifications as adapting the spectral bands of the instrument.

\section{ACKNOWLEDGMENTS}

The authors are thankful for the funding support from the french 'Agence Innovation Défense' (AID).

\section{REFERENCES}

[1] Santamouris, M., "Regulating the damaged thermostat of the cities-Status, impacts and mitigation challenges," Energy and Buildings 91, 43-56 (Mar. 2015).

[2] Mohajerani, A., Bakaric, J., and Jeffrey-Bailey, T., "The urban heat island effect, its causes, and mitigation, with reference to the thermal properties of asphalt concrete," Journal of Environmental Management 197, 522-538 (July 2017).

[3] Lagouarde, J.-P., Bhattacharya, B., Crebassol, P., Gamet, P., Babu, S. S., Boulet, G., Briottet, X., Buddhiraju, K., Cherchali, S., Dadou, I., Dedieu, G., Gouhier, M., Hagolle, O., Irvine, M., Jacob, F., Kumar, A., Kumar, K. K., Laignel, B., Mallick, K., Murthy, C., Olioso, A., Ottle, C., Pandya, M. R., Raju, P. V., Roujean, J.-L., Sekhar, M., Shukla, M. V., Singh, S. K., Sobrino, J., and Ramakrishnan, R., "The Indian-French Trishna Mission: Earth Observation in the Thermal Infrared with High Spatio-Temporal Resolution," in [IGARSS 2018 - 2018 IEEE International Geoscience and Remote Sensing Symposium], 4078-4081, IEEE, Valencia (July 2018).

[4] Poghosyan, A. and Golkar, A., "CubeSat evolution: Analyzing CubeSat capabilities for conducting science missions," Progress in Aerospace Sciences 88, 59-83 (Jan. 2017).

[5] Druart, G., Allioux, R., Perrault, P., Lefranc, V., Cariou, N., and Rousset-Rouvière, L., "Study of infrared optical payloads to be integrated in a nanosat," in [Optical Design and Engineering VII], Mazuray, L., Wartmann, R., and Wood, A. P., eds., 18, SPIE, Frankfurt, Germany (June 2018).

[6] Volatier, J.-B., Duveau, L., and Druart, G., "An exploration of the freeform two-mirror off-axis solution space," Journal of Physics: Photonics 2, 014004 (Dec. 2019).

[7] Lépine, T., Rousselet, N., Surrel, Y., and Houllier, T., "Advanced optical freeform substrates fabricated by ceramic 3D printing and controlled by deflectometry," in [Optical Fabrication, Testing, and Metrology VI], Schröder, S. and Geyl, R., eds., 24, SPIE, Frankfurt, Germany (June 2018).

[8] Zhu, J., Hou, W., Zhang, X., and Jin, G., "Design of a low F-number freeform off-axis three-mirror system with rectangular field-of-view," Journal of Optics 17, 015605 (Jan. 2015).

[9] Thompson, K. P., Fuerschbach, K., Schmid, T., and Rolland, J. P., "Using nodal aberration theory to understand the aberrations of multiple unobscured three mirror anastigmatic (TMA) telescopes," 74330B (Aug. 2009).

[10] Thompson, K. P. and Rolland, J. P., "Freeform Optical Surfaces: A Revolution in Imaging Optical Design," Optics and Photonics News 23, 30 (June 2012). 
[11] Fuerschbach, K., Rolland, J. P., and Thompson, K. P., "A new family of optical systems employing $\varphi$ polynomial surfaces," Optics Express 19, 21919 (Oct. 2011).

[12] Esposito, M. and Zuccaro Marchi, A., "In-orbit demonstration of the first hyperspectral imager for nanosatellites," in [International Conference on Space Optics - ICSO 2018], Karafolas, N., Sodnik, Z., and Cugny, B., eds., 71, SPIE, Chania, Greece (July 2019).

[13] Cakmakci, O., Vo, S., Foroosh, H., and Rolland, J., "Application of radial basis functions to shape description in a dual-element off-axis magnifier," Optics Letters 33, 1237 (June 2008).

[14] Gross, H., Brömel, A., Beier, M., Steinkopf, R., Hartung, J., Zhong, Y., Oleszko, M., and Ochse, D., "Overview on surface representations for freeform surfaces," 96260U (Sept. 2015).

[15] Takaki, N., Bauer, A., and Rolland, J. P., "Degeneracy in freeform surfaces described with orthogonal polynomials," Applied Optics 57, 10348 (Dec. 2018).

[16] Fang, F., Zhang, X., Weckenmann, A., Zhang, G., and Evans, C., "Manufacturing and measurement of freeform optics," CIRP Annals 62(2), 823-846 (2013).

[17] Savio, E., De Chiffre, L., and Schmitt, R., "Metrology of freeform shaped parts," CIRP Annals 56(2), 810-835 (2007). 